\title{
Timing is everything: Early and late neural measures of auditory habituation and discrimination in autism and their relationship to autistic traits and sensory overresponsivity
}

\section{Emily Cary}

Syracuse University https://orcid.org/0000-0003-1354-5395

Devon Pacheco

Syracuse University

Elizabeth Kaplan-Kahn

Syracuse University

Elizabeth McKernan

Syracuse University

\section{Beth Prieve}

Syracuse University

Natalie Russo ( $\square$ nrusso@syr.edu )

Syracuse University https://orcid.org/0000-0001-5229-3552

\section{Research}

Keywords: Autism, brain-behavior correlates, Discrimination, ERPs, Habituation

Posted Date: May 24th, 2021

DOI: https://doi.org/10.21203/rs.3.rs-547935/v1

License: (c) (i) This work is licensed under a Creative Commons Attribution 4.0 International License.

Read Full License 


\section{Abstract}

\section{Background}

Sensory differences are included in the DSM- 5 criteria of autism for the first time, yet it is unclear how sensory behaviors are related to neural indicators of perception. We sought to disentangle this complex relationship by studying early brain signatures of perception using event-related potentials (ERPs) and examining their relationship to sensory overresponsivity and autistic traits.

\section{Methods}

Thirteen autistic children and 13 Typically Developing (TD) children matched on chronological age and nonverbal IQ participated in a passive oddball task, in which P1 habituation and P1 and MMN discrimination were evoked by pure tones. ERPs were compared between groups, and correlations were conducted between ERPs and autistic traits and sensory features.

\section{Results}

Autistic children had marginally enhanced neural discrimination and reduced habituation to auditory stimuli compared to the TD group. Better P1 and MMN discrimination and lower P1 habituation corresponded with more autistic traits. Further, the MMN component, but not P1 components, mapped on to sensory overresponsivity.

\section{Limitations}

Stimuli in the oddball paradigm were not counterbalanced in their presentation as standards or deviants, and participants were not directly asked about their reactions to the auditory stimuli, which would be advantageous in determining whether appraisal of stimuli moderates neural response. The sample size is small and warrants replication.

\section{Conclusions}

Significant correlations between auditory ERP components and autistic traits, even when group differences were not present, suggests benefits to taking a more dimensional approach to autism than using strictly categorical methods. Our findings highlight the significance of temporal and contextual factors in neural information processing as it relates to autistic traits and sensory behaviors.

\section{Background}

Autism is a neurodevelopmental disorder that affects 1 in 54 children ${ }^{1}$ and is characterized by persistent deficits in social communication and interaction, and the presence of restricted, repetitive patterns of behavior, interests, or activities (RRB) ${ }^{2}$. Since the publication of the DSM-5, the RRB criterion of autism includes, for the first time, a focus on sensory differences. While not a diagnostic feature, there is also 
mounting evidence that perception, the interpretation of sensory stimuli, is better or enhanced among autistic individuals across most sensory modalities ${ }^{3,4}$. Enhanced perception in autism includes superior visual discrimination ${ }^{5}$, enhanced low-level auditory perception ${ }^{6,7}$, lower thresholds for vibrotactile stimulation ${ }^{8,9}$, and enhanced olfactory perception ${ }^{10}$.

Evidence is beginning to suggest that perception may be related to sensory differences in autism. In one example, McKernan et al. ${ }^{11}$ found that sensory overresponsivity, defined by atypical responses to sensory stimulation that may include very rapid and intense responses, or prolonged and lasting responses ${ }^{12}$, predicted enhancements in tactile discrimination in autistic individuals. However, whether this finding extends to different sensory modalities (e.g., audition) and measurement types (e.g., neurophysiology) is unknown. The relationship between sensory overresponsivity, or hyperreactivity, and enhanced perception is complicated, as sensory overresponsivity is generally endorsed by parents as reflecting behavioral challenges (e.g., children putting their hands over their ears in response to loud sounds), whereas enhancements in perception are often seen as a source of strength in autism ${ }^{13-15}$. Understanding whether and how sensory behaviors relate to enhanced perception could provide connections linking these disparate areas of study and could have implications for intervention and early diagnoses as both facets are early emerging and predictive of autism ${ }^{16-18}$.

One way to disentangle the complex relationship between perception and sensory features in autism is to look at brain signatures that remain as close as possible, temporally, to the sensory input being processed and map these onto sensory behaviors and autistic traits ${ }^{3,19}$. Specifically, event-related potentials (ERPs), with their millisecond temporal resolution, allow for the examination of two early stages of neurophysiological processes that have been considered atypical in autism and reflective of enhanced perception: discrimination and habituation. Discrimination is the process by which the brain determines that two stimuli differ. It is elicited experimentally using ERPs by comparing the brain's response to a novel stimulus embedded at low rates in the context of a common repetitive stimulus and is commonly measured by the Mismatch Negativity component (MMN) ${ }^{20,21}$. In contrast, habituation reflects the attenuation of an organism's response to a stimulus after repeated exposure and is commonly measured by comparing the neural response to two successive repetitive stimuli using clickedevoked paradigms ${ }^{22}$.

Discrimination and habituation in autism have been measured behaviorally using psychophysical procedures ${ }^{6,23,24}$, and neurophysiologically using ERPs ${ }^{21,25}$ and fMRIs ${ }^{26,27}$ across different sensory modalities and stimulus characteristics (e.g., frequency, duration, intensity). When measured using behavioral or psychophysiological measures in the auditory domain, enhanced discrimination and attenuated habituation are observed in autistic individuals $6,19,25,28-31$ but these findings have not been consistently replicated, likely as a result of differences in measurement, participants' ages and cognitive abilities. Discrepancies may also reflect the heterogeneity of autism, highlighting the probable presence of individual differences ${ }^{22,25,32-37}$. For example, using fMRI methodology, Green et al. ${ }^{38}$ found that over the course of 15 -seconds of simultaneously presented auditory (i.e., traffic noises) and tactile stimuli (i.e., 
scratchy wool), 9 to 17-year-old autistic participants with average IQ habituated less than age and IQ matched typically developing (TD) participants. Decreased habituation was evidenced by both enhanced activation in the amygdala and somatosensory cortices, as well as slow, inconsistent decreases in activity over time. This finding was particularly true for autistic participants and sensory overresponsivity, which was measured by the Sensory Over-Responsivity (SensOR) ${ }^{39}$ scale and specific, modality independent, items from the short Sensory Profile ${ }^{40}$. In contrast, the neural activation of the TD participants quickly and consistently decreased over time. These findings indicate that at least a subset of autistic individuals with higher levels of sensory overresponsivity may also demonstrate altered habituation and discrimination, though given the poor temporal resolution of $\mathrm{fMRI}$, it is difficult to know when in the information processing chain these differences emerged.

Together these findings raise the possibility that early neural indicators of sensory processing might be important to our understanding of the relation between sensory perception and sensory behaviors in autism. However, to-date, ERP studies have generally measured discrimination using components (e.g., P3, MMN) that occur 200 to $300 \mathrm{~ms}$ after the stimuli are presented and compare these to neurophysiological indices of habituation occurring much earlier (e.g., P50). In the present investigation, we examine brain signatures of discrimination and habituation as close as possible, temporally, to the sensory input being processed. To achieve this goal, we leverage a simple and common auditory oddball paradigm in which two trains of stimuli are interleaved, with one being presented $80 \%$ of the time (the standard) and the other presented $20 \%$ of the time (the oddball). Preliminary studies provide promising data for studying habituation in the context of the oddball paradigm by comparing responses to successive standards at the $\mathrm{P} 1{ }^{19}$ or $\mathrm{P} 150{ }^{25}$. Discrimination can be measured in the oddball paradigm by comparing standards and deviants via the MMN. In addition, difference between standards and deviants can also be seen earlier, at the P1, but to our knowledge, this early modulation, which occurs in the same timeframe as the measure of habituation, has never been examined beyond infancy 25 .

Because components with earlier latencies are considered representative of more passive, automatic processing, and later components reflect more cognitive processing ${ }^{41}$, examining the $\mathrm{P} 1$ in addition to the more traditional MMN provides an assessment of more automatic discrimination and could thus be useful in clarifying the links between sensory perception and sensory behaviors in autism ${ }^{42}$. Accordingly, we focus on the early P1 ERP component for habituation, and both the early P1 and later MMN component for discrimination in the same group of autistic children and typically developing children. Further, we examine how these ERP components relate to parent-reported sensory behaviors as well as to autistic traits more broadly for both the TD and autistic participants to elucidate preliminary links between brain and behavior. Only one study to date correlated perception - measured by P1 habituation and MMN discrimination - with the Sensory Profile, specifically focusing on the sensory sensitivity quadrant ${ }^{19}$. They found that TD children (but not autistic children) with larger MMNs had less parentreported sensory sensitivity and that those who habituated less had more typical auditory sensory registration. However, the authors did not match on, nor covary for IQ in their study, and the autistic group had significantly lower IQ than the comparison group (see publications by Russo and Burack ${ }^{43-45}$ for a 
thorough review of the importance of matching). This is problematic for determining whether differences are related to autism or to IQ, and is particularly relevant to studies of auditory processing in autism, as many studies have found that autistic participants with higher IQ often exhibit better discrimination $23,29,31$

Studying differences in neurological sensory processing at the group level, and analyzing individual differences within and across groups as they relate to behavioral indices of autism and sensory processing is key to uncovering broader developmental principles that tie sensation, perception, and autistic features together irrespective of diagnostic label ${ }^{46}$. Here, we take a step towards this goal by correlating electrophysiological measurements of sensory perception with parent-reports of children's autistic traits, measured by the Autism Spectrum Quotient (AQ), and sensory overresponsivity using a subset of relevant items from the Sensory Profile ${ }^{11,38}$. Our aims are to (1) determine if there are differences in ERP markers of habituation and early and late discrimination between autistic and TD children that are matched on age and IQ, (2) determine how autistic traits and sensory overresponsivity relate to early and late neurophysiological indicators of discrimination and habituation, and (3) investigate the relationships between ERP components of discrimination and habituation.

\section{Methods}

\subsection{Participants}

Thirteen autistic children ( 2 females, 11 males) and 13 TD children ( 7 females, 6 males) participated in the study. Autistic and TD children were matched on the basis of chronological age (TD: $M=12.53, S D=$ 2.53; Autism: $M=12.81, S D=2.63$ ) and nonverbal IQ (TD: $M=109.46, S D=10.78$; Autism: $M=110.00, S D$ $=20.48$ ) measured by the Perceptual Reasoning Index (PRI) of the Wechsler Abbreviated Scale of Intelligence Second Edition (WASI-II ${ }^{47}$ ). Autism diagnoses were confirmed by the Autism Diagnostic Observation Schedule Second Edition (ADOS-2 ${ }^{48}$ ) and the Autism Diagnostic Interview-Revised (ADI-R ${ }^{49}$ ) in conjunction with DSM-5 criteria and clinical judgment. All autistic participants met the cut-off for autism as defined by a total score of 7 or higher on the ADOS-2 module $3(n=12)$, and 8 or higher on module $4(n=1)$, as well as clinical judgment. TD participants were those with no other psychological diagnosis. Participants were excluded if they had an IQ below 80 on the WASI-II, or if they had a history of epilepsy, neurological, genetic, psychiatric, or learning disorders. Two autistic participants attempted the auditory oddball task but could not tolerate the stimuli and so the recording was suspended; data from these participants were not included in analyses. 
Table 1

Participant Characteristics

\begin{tabular}{|lllll|}
\hline Component & TD & ASD & $t$ & Sig \\
& M (SD) & M (SD) & & (2-tailed) \\
\hline Age & $12.53(2.53)$ & $12.81(2.63)$ & -0.28 & 0.78 \\
\hline Nonverbal IQ & $109.46(10.78)$ & $110.00(20.48)$ & -0.08 & 0.93 \\
\hline ADOS Severity Score & - & $8.08(1.66)$ & - & - \\
\hline Autism Quotient & $15.08(7.16)$ & $34.00(8.88)$ & $-5.98^{*}$ & $<0.001$ \\
\hline Sensory Overresponsivity & $11.83(9.50)$ & $23.83(8.26)$ & $-3.30 *$ & $<0.01$ \\
\hline Note. Sensory Overresponsivity was calculated using items from the Sensory Profile. \\
\hline
\end{tabular}

\subsection{Stimuli \& Procedure}

Experimental task. The passive oddball paradigm consisted of 1000 trials of pure tones, of which $80 \%$ were standards and $20 \%$ deviants, $1200 \mathrm{~Hz}$ and $1000 \mathrm{~Hz}$, respectively. Stimuli were presented in random order with the exception that two deviants were not played consecutively. The stimulus-onset asynchrony was $600 \mathrm{~ms}$, with each sound presented for $360 \mathrm{~ms}$ with an inter-stimulus interval (ISI) of $240 \mathrm{~ms}$. Participants were seated in a sound-insulated room, and stimuli were presented via two speakers placed to the left and right of the computer screen at an intensity of $60 \mathrm{~dB}$ sound-pressure level. A visual distraction task was used, in which participants were instructed to watch a silent subtitled movie or television show of their choice, while ignoring the sounds, as these are optimal conditions to eliminate other cognitive components (e.g., the N200 and P300) that are active during attention ${ }^{50}$.

Procedure. The study was approved by the local Institutional Review Board, and all parents and children signed consent and assent forms, respectively, prior to taking part in the study. Participants were compensated $\$ 10$ for every hour of participation. All participants had their hearing evaluated at the Pediatric Audiology Laboratory at Syracuse University. Participants completed the audiological evaluation and EEG assessments in counterbalanced order. The audiology assessments included otoscopy, behavioral audiometric threshold evaluation from $.25-8 \mathrm{~Hz}$, including inter-octaves 3 and 6 $\mathrm{kHz}$, distortion product otoacoustic emissions (DPOAEs) from 1.5-8 kHz (f2:f1 = 1.22; L1:L2 = $10 \mathrm{~dB} ; \mathrm{L} 2=$ $55 \mathrm{~dB}$ SPL) transient click evoked otoacoustic emissions, and wide band absorbance. In cases when standard behavioral audiometric testing could not be completed, conditioned play audiometry was utilized. Hearing was considered to be normal if the behavioral thresholds were $<25 \mathrm{~dB} H \mathrm{HL}$. Following the hearing evaluation, speech and click evoked auditory brainstem responses were recorded monaurally and binaurally from each participant. Speech evoked auditory brainstem responses were collected to a $40 \mathrm{~ms}$ /da/ stimuli at $63 \mathrm{~dB} \mathrm{nHL}$ and click evoked ABR were evoked using a $100 \mu$ s click at $70 \mathrm{~dB} \mathrm{nHL}$ (though are not presented here). 
While the children participated in the experimental tasks, their parents filled out the Sensory Profile ${ }^{40}$ and the Autism Spectrum Quotient $\left(A Q{ }^{51}\right)$. Participants completed the WASI-II and ADOS-2 at a separate lab visit.

\subsection{Measures}

Sensory Profile ${ }^{40}$. The Sensory Profile is a 125 -item questionnaire completed by a parent that measures the extent to which sensory processing contributes to children's behavior. While not a specific scale on the Sensory Profile, based on the work of others ${ }^{38}$ we calculated a sensory overresponsivity composite from the sum of 14 items that capture tactile sensitivity, auditory filtering, and visual/auditory sensitivity (see McKernan et al. ${ }^{11}$ for specific items). Items were reverse scored, such that higher scores indicate more sensory overresponsivity.

The Sensory Profile has good internal consistency using Cronbach's alpha, with subscale coefficients ranging from 0.47 to $0.91^{40}$. There is preliminary evidence of content and construct validity, as well as convergent and discriminant validity, evidenced through its comparison with the School Function Assessment and through differentiating among diagnostic groups (e.g., differentiating autistic from TD children ${ }^{40,52}$ ).

Autism Spectrum Quotient ( $A Q{ }^{51,53}$ ). Three forms of the $A Q$ were administered depending on the participant's age; the parent-report Child $A Q$ for ages 4-11, Adolescent $A Q$ for ages $12-15^{53}$, and the self-report Adult $A Q$ for participants 16 and older ${ }^{51}$. All questionnaires contain 50 items, with item content consistent across forms, but adapted for the developmental level. Items (e.g., "s/he often notices small sounds when others do not") are rated on a 4-point scale from "definitely agree" to "definitely disagree", with approximately half of items reverse-scored. Items on the AQ were collapsed across the 4 response options and dichotomously scored (i.e., 0 and 1) to get a total $A Q$ score range of 0 to $5051(p), 53$, with higher scores indicating a higher presence of autistic traits, including poor social skills, poor communication skills, poor imagination, exceptional attention to detail, and poor attention-switching. A score of 32 or higher is highly predictive of autism. The total score was used in this study as a measure of autistic traits.

Evidence of validity for the $A Q$ includes its predictive ability to identify who receives an autism diagnosis in clinics and good discriminant and convergent validity ${ }^{54}$. The $A Q$ also has good test-retest and interrater reliability ${ }^{51}$.

Wechsler Abbreviated Scale of Intelligence, Second Edition (WASI-II; Wechsler, 2011). The WASI-II is an abbreviated measure of cognitive intelligence for individuals 6 to 90 years of age. For this study autistic participants were matched with typically developing participants using the Perceptual Reasoning (PRI) domain of the WASI-II, a measure of nonverbal IQ. The PRI is composed of two subscales that include block design and matrix reasoning.

\subsection{Recording and Analysis of ERP Waveforms}


ERPs were recorded with a high-density 128-channel Geodesic SensorNet using the NetStation platform, and a sampling rate of $1024 \mathrm{~Hz}$. Data were filtered between $1 \mathrm{~Hz}$ and $30 \mathrm{~Hz}$ using a Butterworth filter, referenced to $\mathrm{Cz}$ during acquisition. Data were re-referenced to the mastoids during offline processing, and ERP segments were extracted - 50 to $650 \mathrm{~ms}$ centered around the stimulus onset. Data were baseline corrected from - 50 to $0 \mathrm{~ms}$. After epochs containing artifacts greater than 100 microvolts $(\mu \mathrm{V})$ were removed, ERP waves were visually inspected, and bad channels were interpolated using spline interpolation techniques ${ }^{55}$. Post-acquisition processing and data extraction were completed using the EEGLAB v14.1.2 ${ }^{56}$ and ERPLAB v7.0.0 ${ }^{57}$ toolboxes in MATLAB R2017a.

The ERP trials for each individual were sorted into four bins that allowed for the measurement of habituation and discrimination. These included: (1) 1st standard, which reflects the ERPs time locked to the onset of the first standard following a deviant; (2) 2nd standard, which reflects the ERPs time locked to the onset of the second standard following a deviant (Fig. 1A); (3) all standards and (4) all deviants. Trials were averaged for each participant for each bin. For group-based comparisons, separate grand averages were computed for the TD and autism participants, while for the individual difference analyses, the data were not collapsed by group.

P1. To determine the time window for measurement of P1 amplitude, the ERPs evoked by the standard and deviant stimuli were averaged together and collapsed across groups in order to identify one time window that does not rely on our dependent variables, namely condition and group (Russo et al., 2010). The peak amplitude of the resultant waveform was measured and a $50 \mathrm{~ms}$ window was created around that peak. The P1 peak of the averaged waveform occurred at $96 \mathrm{~ms}$, and accordingly, the P1 amplitude was measured as the mean amplitude between 71 and $121 \mathrm{~ms}$ at the FCz electrode (number 6 in EGI layout). P1 peak latency was calculated in individual waveforms as the positive peak from 50 to $150 \mathrm{~ms}$ at the FCz electrode. This wider range was used to capture variability in P1 latencies and to enhance specificity for the analysis of individual differences ${ }^{59}$.

P1 habituation was calculated by subtracting each participants' averaged 2nd standard waveform from the average waveform to the 1 st standard. Similarly, P1 discrimination was calculated by subtracting the average waveform evoked by all standard stimuli from that evoked by the deviant stimuli for each participant. On the basis of these difference waves, mean amplitudes and peak latencies were calculated.

MMN. The MMN was calculated by subtracting each participant's deviant waveform from their standard waveform, and the average latency was identified when waveforms were collapsed across participant groups. Based on research indicating that the MMN typically peaks from 150 to $250 \mathrm{~ms}$ post-stimulus, with maximum amplitude in the fronto-central midline ${ }^{20,21}, \mathrm{MMN}$ was measured as the mean amplitude of the most negative peak in that range at the FCz electrode. The amplitude of the MMN peak was -2.58 $\mu \mathrm{V}$, which occurred at a latency of $176 \mathrm{~ms}$. Consequently, MMN amplitude was measured as the mean amplitude between 126 to $226 \mathrm{~ms}$, a $50 \mathrm{~ms}$ range around the peak latency. Whereas peak latency is often appropriate for small distinct early components such as the $\mathrm{P} 1{ }^{60,61}$, the fractional-area latency method was chosen to measure latency in the MMN because this method is considered to be more reliable and 
less sensitive to noise than peak latency, especially for large components ${ }^{20}$. Fractional-area latency was measured as the time in milliseconds at which $50 \%$ of the area under the curve has been accrued, capturing the latency of the negative peak between 126 and $226 \mathrm{~ms}$.

\section{Results}

Data were analyzed and graphed using IBM SPSS Statistics for Windows, version 26, and EEGLAB v14.1.2 ${ }^{56}$ and ERPLAB v7.0.0 ${ }^{57}$ toolboxes in MATLAB R2017a.

\subsection{Hearing}

Audiological results from four participants were considered to be outside the range of normal. In one participant who was TD, the behavioral threshold at $.250 \mathrm{kHz}$ was $25 \mathrm{~dB} \mathrm{HL}$ and in one autistic participant, the behavioral threshold at $8 \mathrm{kHz}$ was $25 \mathrm{~dB} \mathrm{HL}$. One participant who was TD had abnormal middle ear function in the left ear and elevated thresholds at 2,000 and $4,000 \mathrm{~Hz}$, and one autistic participant had abnormal middle ear function in the left ear and elevated thresholds at $250 \mathrm{~Hz}$ and 6,000 $\mathrm{Hz}$. The data from these participants were included in the analysis because none of these participants had elevated thresholds at the frequencies of the stimuli and their ERP waveforms did not deviate from group norms. The rest of the participants had normal hearing, distortion product and transient evoked otoacoustic emissions, and wideband absorbance.

\subsection{ERP Descriptive Information}

Grand average waveforms comparing the 1st (solid line) and 2nd (dotted line) standard for the TD (black lines) and the autistic (grey lines) participants are presented in panel A of Fig. 1. Panel B illustrates grand averages for the TD (black lines) and autistic (grey lines) participants for standard (solid lines) and deviant (dotted line) stimuli. In both panels $A$ and B, there is a clear positive P1 peak around $100 \mathrm{~ms}$ (indicated by the arrow). Grand average differences waves of standards and deviants for the TD (black lines) and autistic (grey lines) participants are presented in panel C. A negativity was noted between 150 and $250 \mathrm{~ms}$ reflecting the typical time course and morphology of the MMN ${ }^{21}$. While not in our original hypotheses, visual inspection of the MMN latency appeared to differ by group and was thus included in our analyses post hoc. Approximately $26 \%$ of each trial type were rejected. The average number of accepted trials per participant were 744, with 613 standards, 131 deviants, 130 1st standards, and 103 2 nd standards. The number of accepted trials did not differ by group $(p=.90)$.

Figure 1. Response-locked ERP waveforms at the FCz electrode site for Autism Spectrum Disorder (ASD) and TD children during the oddball task, corresponding to (A) P1 habituation, comparing the 1st to 2nd successive standard, (B) P1 discrimination, comparing the standard to the deviant stimuli, and (C) MMN discrimination from subtracting the standard from the deviant grand average waveforms.

\subsection{Group Differences in sensory responsivity, P1 Habituation and P1 and MMN Discrimination}


As expected, AQ scores and sensory overresponsivity scores extracted from the Sensory Profile were significantly higher for the autism group than the TD group, indicating higher autistic traits and more intense and/or prolonged responses to sensory stimulation (see Table 1).

As depicted in Table 2, independent samples t-tests were conducted between autism and TD groups for (1) P1 habituation, (2) P1 discrimination, and (3) MMN discrimination.

Table 2

Independent Samples T-test, comparing ASD to TD participants $(\mathrm{df}=24)$

\begin{tabular}{|lllll|}
\hline ERP Amplitude & TD & ASD & $t$ & Sig \\
& M (SD) & M (SD) & & (2-tailed) \\
\hline P1 habituation & $0.92(1.19)$ & $-0.71(1.76)$ & $2.76^{\star}$ & 0.01 \\
\hline P1 discrimination & $-0.40(1.04)$ & $-1.23(1.34)$ & $1.76^{\dagger}$ & 0.09 \\
\hline MMN discrimination & $-1.72(1.41)$ & $-2.81(2.17)$ & 1.51 & 0.15 \\
\hline${ }^{\dagger} p<0.1{ }^{*} p<0.05$ significance & & & \\
\hline
\end{tabular}

Autistic participants habituated to successive stimuli significantly less than TD participants. In fact, while the TD participants habituated as expected, as evidenced by a larger P1 amplitude to the 1st standard relative to the 2 nd standard, the opposite pattern occurred for the autistic participants, with the amplitude of the P1 to the 2nd standard being higher than the amplitude of the P1 to the 1st standard (Fig. 2). Autistic participants discriminated marginally, though not significantly, more than TD participants at the P1 component (Fig. 2) when standards were contrasted with deviants. There were no differences in discrimination for the MMN component, but autistic participants had marginally, though not significantly, earlier MMN latencies than TD participants.

Figure 2. P1 habituation (amplitude of 1st minus 2nd standard) and P1 discrimination (amplitude of deviant minus standard) analyzed by group $(95 \% \mathrm{Cl})$.

\subsection{Relation between Early and Later Processing of Discrimination}

We hypothesized that the P1 discrimination and the MMN, would be correlated with one another, as they both measure discrimination. Conversely, we anticipated that dissimilar constructs (habituation and both discrimination measures) would not be correlated. Given that the P1 discrimination measure has not been previously used in the literature this would provide some assurance of the validity of this novel measure. P1 habituation was not significantly correlated with the $\mathrm{MMN}(r(24)=0.28, p=.17)$, nor was P1 discrimination correlated with P1 habituation $(r(24)=0.22, p=.29)$. In contrast, P1 discrimination correlated highly with the $\mathrm{MMN}$ discrimination $(r(24)=0.64, p<.001)$, as illustrated in Fig. 3. Interestingly, and exploratorily, when analyzed by group, in TD participants, P1 discrimination was highly associated 
with $\mathrm{MMN}$ discrimination $(r(11)=0.82, p=.001)$, but only marginally, and not significantly, associated with the MMN discrimination for autistic participants $(r(11)=0.49, p=.09)$.

Figure 3. A significant positive correlation between the MMN amplitude and P1 discrimination amplitude.

\subsection{Correlations between Autistic Traits and P1 Habituation and P1 and MMN Discrimination}

Preliminary correlations were conducted with age and PRI to determine whether these should be covaried. For the MMN only, latency was correlated with age $(r(24)=-0.40, p=.05)$ indicating that older children had earlier MMN latencies. Age was thus controlled for in correlations centered on MMN latency.

Total scores on the AQ were significantly correlated with several measures that included $\mathrm{P} 1$ habituation amplitude $(r(24)=-0.38, p=.05), \mathrm{P} 1$ discrimination amplitude $(r(24)=-0.38, p=.06)$, and MMN amplitudes $(r(24)=-0.49, p=.01)$. Overall, higher autistic traits were associated with decreased (or reversed) habituation (Fig. 4A) as well as greater (early and late) discrimination (Fig. 4B).

Total AQ scores were also correlated with $\mathrm{P} 1$ and $\mathrm{MMN}$ latencies such that those with higher $\mathrm{AQ}$ scores had later P1 discrimination latencies $(r(24)=0.41, p=.04)$ but marginally earlier MMN latencies $(r(24)=$ $-0.36, p=.08)$. The latency of P1 habituation was unrelated to autistic traits.

Table 3

Correlations between measures of autistic traits and P1 and MMN Amplitudes

\begin{tabular}{|llll|}
\hline & P1 habituation & P1 discrimination & MMN discrimination \\
\hline Autism Quotient & $-0.38^{*}$ & $-0.38^{\dagger}$ & $-0.49^{*}$ \\
\hline Sensory Overresponsivity & -0.15 & -0.32 & $-0.49^{*}$ \\
\hline Note. Sensory Overresponsivity was calculated using items from the Sensory Profile. \\
\hline${ }^{\dagger} p<0.1,{ }^{*} p<0.05$ significance & & \\
\hline
\end{tabular}

Whereas P1 indices of habituation and discrimination did not correlate with sensory overresponsivity, higher MMN amplitude was significantly associated with higher sensory overresponsivity $(r(22)=-0.49, p$ $=.02)$ (Fig. 4C).

Figure 4. Correlations between the $\mathrm{AQ}$ and $\mathrm{A}$ ) $\mathrm{P} 1$ habituation amplitude and $\mathrm{B}$ ) $\mathrm{MMN}$ amplitude, and $\mathrm{C}$ ) between Sensory Overresponsivity and the MMN, by group.

\section{Discussion}

The goals of the current study were to test for group differences between neural measures of habituation and discrimination between TD and autistic children, and to assess the relationship between 
neurophysiological indicators and parent-reported autistic traits and sensory features as a means of bridging the gap between our understanding of sensation and perception and behavior and brain in autism. Our main findings were that neurophysiological indices of habituation measured in the first $100 \mathrm{~ms}$ of processing differed between autistic and TD children. While TD children showed the anticipated pattern of decreases in neuronal response between the first and second presentation of a stimulus in sequence, autistic children of the same age and cognitive ability showed the opposite pattern of having a greater neural response to the second of two stimuli. For discrimination, marginally significant group differences were noted between those with and without autism within the first $100 \mathrm{~ms}$ of processing such that autistic children of the same age and cognitive ability as the TD children had a greater difference in their neural responses to standards and deviants than TD peers. With respect to brain and behavior relationships, we found that habituation amplitude was inversely related to autistic traits and that individual differences in amplitudes of the MMN were linked to both autistic traits and to sensory overresponsivity, with greater discrimination being linked to both higher traits and higher overresponsivity as measured by parent report. While exploratory, we also found that P1 and MMN indices of discrimination were highly correlated in TD children and were decoupled, or uncorrelated, in autistic individuals. Together, these findings suggest the presence of early neurophysiological differences in auditory processing that indicate enhanced discrimination and decreased habituation in autism and that these map on to autistic features.

\subsection{P1 Habituation}

Habituation measured in the first $100 \mathrm{~ms}$ of processing differed significantly between autistic and TD children, which is consistent with literature measuring habituation during an oddball paradigm ${ }^{19,25,28}$. However, sensory gating studies that are more traditionally associated with habituation find either no differences ${ }^{32}$, or reduced P50 gating in autism. A recent meta-analysis of sensory gating in autism synthesized that the reduction in sensory gating in autism is driven by a smaller response to the first stimulus in a pair, not the second, and therefore may not accurately reflect a reduction in the autistic brain's ability to filter out information ${ }^{62}$. In contrast, when habituation is measured in the context of an oddball paradigm, reductions in habituation are consistently noted ${ }^{19,25,28}$. We find this same trend in our study, with responses of the TD and autistic participants being similar in amplitude for the first stimulus, but larger in amplitude for the second stimulus in autism. These findings suggest altered modulation of incoming auditory stimuli and importantly, because groups were matched on age and IQ, we can rule out developmental and cognitive differences as a confound.

We offer several ways to reconcile differences between paradigms purported to measure the same things. First, differences might be due to effects of context. In sensory gating paradigms, stimuli are presented in pairs of clicks, with a clear temporal demarcation of time between sets of pairs, or trials. That is, the first auditory stimulus that is delivered activates inhibitory gating mechanisms that are responsible for diminishing the response to the second stimulus ${ }^{63}$. In contrast, in oddball paradigms, we are looking at habituation within a broad context of continuous stimuli without a clear temporal reset. As such, in 
oddball paradigms, the "gate" is opened by the first stimulus in the block and does not reset. We propose that habituation, measured within an oddball paradigm, might be more reflective of how the brain copes with repetitive streams of information and might be more relevant to our understanding of autism. This interpretation, which needs to be tested empirically, could help explain discrepancies in findings for these two measures of habituation.

\subsection{P1 Discrimination}

In general, the P1 is an exogenous ERP component modulated by stimulus characteristics. For example, in the visual domain brighter stimuli elicit larger P1s than stimuli that are dimmer ${ }^{64}$ and in the auditory domain, louder sounds elicit larger early ERPs than quieter sounds ${ }^{65,66}$. In the present study, we find that the $\mathrm{P} 1$ was modulated by frequency, such that the higher frequency standards $(1200 \mathrm{~Hz})$ yielded larger $\mathrm{P} 1$ amplitudes than the lower frequency deviants $(1000 \mathrm{~Hz})$. This finding suggests that the paradigm was sensitive enough to elicit differences in exogenous ERPs overall. In addition, one novel aspect of this P1 sensory discrimination measure was that we computed a difference wave, analogous to both the P1 habituation measure as well as the MMN. To provide some validity of this component, we computed correlations with the expectation that measures of discrimination should be more related to each other (e.g., P1 discrimination and MMN) than to habituation measures (e.g., the two P1 difference waves). P1discrimination and MMN amplitudes were highly correlated overall and were uncorrelated with the P1 habituation measure. Though preliminary and in need of replication, this P1 discrimination difference wave could be useful to examine across stimulus characteristics and sensory modalities to determine if sensory modulation, rather than absolute amplitudes, differ between those with and without autism.

The P1 was modulated by stimulus characteristics overall, and marginally differed between groups, such that autistic children had slightly larger P1 difference waves than TD participants. This provides preliminary evidence for the presence of enhancements in early perceptual markers of discrimination. Using a similar oddball paradigm and difference wave, Guiraud et al. ${ }^{25}$ examined discrimination to auditory deviants and standard sounds and compared the P150 ERP component between low and highrisk infants defined by virtue of having an autistic sibling. They found that infants at high risk for autism did not differ between standard and deviants, whereas low-risk infants did, as indicated by a greater P150 to deviants than standards. While our findings trend in the opposite direction to this singular study, the development of early auditory ERPs is significant ${ }^{67-69}$ making comparisons difficult. Further work in this area is warranted to both substantiate the trends in our data, as well as to examine whether these extend to other stimulus characteristics (e.g., duration) and different frequencies (e.g., pure tones vs complex tones $\left.{ }^{6,7,70}\right)$.

\subsection{MMN}

The $M M N$, a measure of pre-attentive discrimination, has long been thought of as a potential prognostic indicator of autism, as a result of its relationship to the development of language ${ }^{71,72}$. While many studies of the MMN have been conducted with this population ${ }^{73,74}$, there has been little consensus overall, with several studies findings larger, smaller or equivalent MMN amplitudes and latencies between 
those with and without autism. Here we find that MMN amplitudes did not differ between those with and without autism. While on the surface this seems inconsistent with a variety of other studies, a careful consideration of stimulus parameters yields a remarkably consistent story. Specifically, studies that measured the MMN via changes in frequency of pure tones, rather than complex tones or speech, almost all found no differences between groups ${ }^{28,37,75-78}$ suggesting a core role of stimulus complexity in discrimination ${ }^{79}$ in autism. There were only two studies that found group differences in MMN amplitudes for pure tones ${ }^{19,80}$. Notably, in both of these studies the autistic individuals also had concurrent intellectual disability or IQ scores significantly below that of the TD participants. Intellectual ability may modulate discrimination, as autistic individuals and higher IQs often exhibit better discrimination 23,29,31. Together, these findings speak to the importance of specificity in stimulus selection ${ }^{73,74}$ and mental age matching strategies ${ }^{43-45}$ as they relate to our understanding of the auditory discrimination and the MMN in autism.

In addition to finding subtle methodological and experimental differences (e.g., stimulus complexity; cognitive ability) that can explain the heterogeneity of group-based research findings, one must also consider that group means, which serve as the dependent variable in these types of designs might not capture or represent the heterogeneity of autism. Given that on average most MMN studies (including ours) have small sample sizes (e.g., around 15 participants), the influence of any one participant is mathematically larger than in cases where large samples are available. One way to embrace this heterogeneity is to focus on individual differences by examining brain-behavior correlations. This allows us to move beyond group comparisons to a more dimensional understanding of how specific brain signatures relate might map onto behavioral parent-reported characteristics, agnostic to diagnostic label.

\subsection{Brain-behavior correlates}

There were two main goals in analyzing brain behavior relationship in the present study. One goal was to examine how different measures of habituation and discrimination mapped on to autistic traits. A second aim was to analyze individual differences in sensory processing to help bridge the gap between sensation and perception by studying how brain signatures occurring closer (e.g., P1) and later (e.g., $\mathrm{MMN}$ ) temporally to the onset of the sensory stimulus relate to sensory behaviors. In the present study the amplitudes for all three of the ERP components were correlated with autistic traits. Specifically, larger discrimination and smaller habituation amplitudes were related to higher levels of autistic traits across the entire sample of TD and autistic participants. Additionally, findings that individuals with higher levels of autistic traits had later P1 and earlier MMN latencies are in line with research demonstrating that autistic individuals rely more on early perceptual processing, which then impacts subsequent processing (Russo et al., 2012). Together, these findings suggest that the AQ captures some aspects of autism that are related to neurophysiological markers of auditory processing and suggest continued use of the $A Q$ as a correlate $51,82,83$. Surprisingly, we found that late, but not early ERP components that included P1 discrimination and habituation, were related to sensory behaviors as measured by overresponsivity. 
In general ERP components with later latencies reflect more endogenous cognitively driven, top down influences, whereas earlier ERPs tend to reflect automatic perception ${ }^{41}$. This heuristic can help contextualize the lack of a significant correlation between early ERPs and parent-reported measures of sensory overresponsivity and suggests that temporal factors might be critical to our understanding of the brain behavior relationship in autism. The P1 discrimination measure used here is novel and therefore we rely on previous research focused on early indicators of habitation to develop hypotheses that we are tentatively generalizing to the other $\mathrm{P} 1$ component.

The lack of correlation between sensory features and P1 ERP amplitudes replicates the findings of others ${ }^{19}$ but differs from general findings that less, or slower, habituation in autistic participants is associated with an increase in sensory behaviors ${ }^{28,38}$. One pivotal difference that could account for these discrepant findings relates to the timescale of measurements. For example, the relation between habitation and sensory overresponsivity noted by Green occurred in the context of an fMRI study - which by definition has poor temporal resolution - and over the course of 15-second blocks. Further, Hudac et al. ${ }^{28}$ measured habituation by looking at amplitude attenuation of the N1 and P3 ERP components over the course of several minutes (approximately 80 trials) and found that slower habituation to novel, nonrepeating sounds, correlated with sensory seeking behaviors at the P3 but not at N1. That is, when measured by looking at early, exogenous ERP components, few relationships between habituation and sensory features are noted. However, when measured through endogenous, late ERP components (e.g., P3) or with fMRI these brain behavior relationships begin to emerge. Together, these findings suggest that temporal scales, as they relate to when in time one looks (e.g., early or late ERP components) as well as the length of time over which measurements are made (e.g., milliseconds vs. several seconds) might not only be critical to our understanding of habituation in autism, but also more broadly to the relationship between information processing and sensory behaviors in autism.

\subsection{Relation between Early and Later Processing of Discrimination}

Exploratory correlational analyses supported the hypothesis that early processes of discrimination (P1) would correlate with later measures of discrimination ( $M M N)$ when analyzed using the whole sample. While this was true of the group overall, this was not the case when we examined the two groups separately. Specifically, for TD participants, P1 discrimination was highly associated with MMN discrimination. However, this correlation was only marginally present for autistic participants. While the samples are small and the analyses are exploratory this lack of relationship in the autism group support Mottron's EPF model that low-level perception might operate more independently in autistic cognition than in typical development ${ }^{3}$.

\section{Limitations}

One limitation of this study is that the stimuli in the oddball paradigm were not counterbalanced and stimulus characteristics may modulate the $\mathrm{P} 1$ and $\mathrm{MMN}$ 65,66,74,84. As such, it may be that participants ERP waves varied as a product of the tone, rather than the categorization as a standard or deviant 
stimulus. Second, participants were not directly asked about their thoughts and reactions to the auditory stimuli used in this study, which would be advantageous in determining whether one's appraisal of stimuli moderates their neural response. For example, the two prospective participants who did not participate in the study because they found the sounds intolerable to listen to suggests that the stimuli was more bothersome for some participants than others, and this variation in arousal can moderate components including the $\mathrm{P} 1{ }^{20}$. Third, we did not direct participants attention to the auditory stimuli and as such are measuring only passive processing, which could be particularly relevant to our understanding of sensation and perception in autism (e.g., $\left.{ }^{85,86}\right)$.

A notable limitation is the small sample size, and thus effects are in need of replication, especially with respect to individual differences ${ }^{87}$. Additionally, alpha was set to 0.05 for analyses and accordingly these results should be interpreted carefully as analyses may include false positives.

\section{Conclusions}

Our results suggest that autistic children have enhanced neural discrimination and reduced habituation to auditory stimuli, and that these neural indicators map onto autistic traits. Further, the late ERP components but not early ones mapped on to sensory overresponsivity. In both these cases better discrimination and lower habituation were related to core features of autism. Findings of significant correlations between auditory ERP components and autistic traits, even when group differences were not present, suggests that taking a more dimensional approach to autism allows for clarifying questions left unanswered when using strictly categorical methods.

Our findings highlight the significance of contextual and temporal factors in information processing and its relationship to autistic traits and sensory behaviors. The clinical utility of understanding the context (e.g., stimulus complexity, characteristics), temporal nature (e.g., a concise pair of stimuli or an ongoing train of stimuli), and temporal scales (e.g., when in time one looks; length of time measurements are made) of information processing, is that we can then better understand which contexts are linked to sensory behaviors that are reported as causing distress and make environmental adaptations to reduce discomfort. Accordingly, environmental accommodations related to sensory overresponsivity might be useful, including developmentally appropriate sensory-motor experiences and structured physical and sensory environments ${ }^{88}$.

\section{Declarations}

\section{Ethics approval and consent to participate}

The study was approved by the local Institutional Review Board, and all parents and children signed consent and assent forms, respectively, prior to taking part in the study.

\section{Consent for publication}


Not applicable

\section{Availability of data and materials}

The de-identified dataset used during the current study are available from the corresponding author on reasonable request.

\section{Competing Interests}

The authors declare that they have no competing interests

\section{Funding}

This work was supported by the Collaboration for Unprecedented Success and Excellence (CUSE) Grant to N.R. and B.P. and the National Institute for Mental Health (NIMH) funding (grant number MH101536) to N.R.

\section{Author's Contributions}

EC collected and analyzed the data, wrote the manuscript. DV collected and analyzed the audiology data. BP collaborated with the study conceptualization. EKK collected clinical data, and assisted with ERP analysis. EM collected clinical data. NR conceptualized the study, experimental design, and collaborated in the writing of the final manuscript. All authors read and approved the final manuscript.

\section{Acknowledgements}

We thank Dr. Justin Kopec, Dr. Nicole Shea, Margie Garber, Alyssa Prawl, and Angela Madrid for their assistance with data collection, and Ellie Masters for assistance with data analysis. We thank the children and their families for participating in our research.

\section{Footnotes}

We respect the right for autistic people to choose whether they prefer identity- or person-first language. We use identity-first language based on the preferences that the majority of autistic individuals endorse and also acknowledge that others prefer person-first language. For these same reasons and for simplification, we also use the term autism to refer to the overall diagnostic category in the DSM-5 called autism spectrum disorder (ASD). However, we use the term ASD in the figures and plots for brevity.

\section{References}

1. Maenner MJ, Shaw KA, Baio J, et al. Prevalence of Autism Spectrum Disorder Among Children Aged 8 Years - Autism and Developmental Disabilities Monitoring Network, 11 Sites, United States, 2016. MMWR Surveill Summ. 2020;69(4):1-12. doi:10.15585/mmwr.ss6904a1 
2. American Psychiatric Association. Diagnostic and Statistical Manual of Mental Disorders. American Psychiatric Association; 2013.

3. Mottron L, Burack JA. Enhanced perceptual functioning in the development of autism. In: The Development of Autism: Perspectives from Theory and Research. Lawrence Erlbaum Associates Publishers; 2001:131-148.

4. Mottron L, Dawson M, Soulières I, Hubert B, Burack J. Enhanced Perceptual Functioning in Autism: An Update, and Eight Principles of Autistic Perception. J Autism Dev Disord. 2006;36(1):27-43. doi:10.1007/s10803-005-0040-7

5. Dakin S, Frith U. Vagaries of Visual Perception in Autism. Neuron. 2005;48(3):497-507. doi:10.1016/j.neuron.2005.10.018

6. Bonnel A, McAdams S, Smith B, et al. Enhanced pure-tone pitch discrimination among persons with autism but not Asperger syndrome. Neuropsychologia. 2010;48(9):2465-2475. doi:10.1016/j.neuropsychologia.2010.04.020

7. Samson F, Mottron L, Jemel B, Belin P, Ciocca V. Can Spectro-Temporal Complexity Explain the Autistic Pattern of Performance on Auditory Tasks? J Autism Dev Disord. 2006;36(1):65-76. doi:10.1007/s10803-005-0043-4

8. Blakemore S-J, Tavassoli T, Calò S, et al. Tactile sensitivity in Asperger syndrome. Brain Cogn. 2006;61(1):5-13. doi:10.1016/j.bandc.2005.12.013

9. Cascio C, McGlone F, Folger S, et al. Tactile Perception in Adults with Autism: a Multidimensional Psychophysical Study. J Autism Dev Disord. 2008;38(1):127-137. doi:10.1007/s10803-007-0370-8

10. Ashwin C, Chapman E, Howells J, Rhydderch D, Walker I, Baron-Cohen S. Enhanced olfactory sensitivity in autism spectrum conditions. Mol Autism. 2014;5(1):53. doi:10.1186/2040-2392-5-53

11. McKernan EP, Wu Y, Russo N. Sensory Overresponsivity as a Predictor of Amplitude Discrimination Performance in Youth with ASD. J Autism Dev Disord. Published online April 23, 2019. doi:10.1007/s10803-019-04013-0

12. Ben-Sasson A, Hen L, Fluss R, Cermak SA, Engel-Yeger B, Gal E. A Meta-Analysis of Sensory Modulation Symptoms in Individuals with Autism Spectrum Disorders. J Autism Dev Disord. 2009;39(1):1-11. doi:10.1007/s10803-008-0593-3

13. Mottron L. Detrimental Sensitivity" framework misses the positive performance, role and autonomy of autistic perception. Cogn Neurosci. 2019;10(3):168-169. doi:10.1080/17588928.2019.1596073

14. Hagmann CE, Wyble B, Shea N, LeBlanc M, Kates WR, Russo N. Children with autism detect targets at very rapid presentation rates with similar accuracy as adults. J Autism Dev Disord. 2016;46(5):17621772. doi:10.1007/s10803-016-2705-9

15. Kopec J, Hagmann C, Shea N, Prawl A, Batkin D, Russo N. Examining the Temporal Limits of Enhanced Visual Feature Detection in Children With Autism. Autism Res. 2020;13(9):1561-1572. doi:10.1002/aur.2361

16. Boyd BA, Baranek GT, Sideris J, et al. Sensory features and repetitive behaviors in children with autism and developmental delays. Autism Res. 2010;3(2):78-87. doi:https://doi.org/10.1002/aur.124 
17. Gliga T, Bedford R, Charman T, et al. Enhanced Visual Search in Infancy Predicts Emerging Autism Symptoms. Curr Biol. 2015;25(13):1727-1730. doi:10.1016/j.cub.2015.05.011

18. Robertson CE, Baron-Cohen S. Sensory perception in autism. Nat Rev Neurosci. 2017;18(11):671-684. doi:10.1038/nrn.2017.112

19. Ruiz-Martínez FJ, Rodríguez-Martínez El, Wilson CE, Yau S, Saldaña D, Gómez CM. Impaired P1 Habituation and Mismatch Negativity in Children with Autism Spectrum Disorder. J Autism Dev Disord. 2020;50(2):603-616. doi:10.1007/s10803-019-04299-0

20. Luck SJ. An Introduction to the Event-Related Potential Technique. Second edition. The MIT Press; 2014.

21. Näätänen R, Paavilainen P, Rinne T, Alho K. The mismatch negativity (MMN) in basic research of central auditory processing: A review. Clin Neurophysiol. 2007;118(12):2544-2590.

doi:10.1016/j.clinph.2007.04.026

22. Orekhova EV, Stroganova TA, Prokofyev AO, Nygren G, Gillberg C, Elam M. Sensory gating in young children with autism: Relation to age, IQ, and EEG gamma oscillations. Neurosci Lett. 2008;434(2):218-223. doi:10.1016/j.neulet.2008.01.066

23. Jones CRG, Happé F, Baird G, et al. Auditory discrimination and auditory sensory behaviours in autism spectrum disorders. Neuropsychologia. 2009;47(13):2850-2858. doi:10.1016/j.neuropsychologia.2009.06.015

24. Mottron L, Peretz I, Ménard E. Local and Global Processing of Music in High-functioning Persons with Autism: Beyond Central Coherence? J Child Psychol Psychiatry. 2000;41(8):1057-1065. doi:https://doi.org/10.1111/1469-7610.00693

25. Guiraud JA, Kushnerenko E, Tomalski P, Davies K, Ribeiro H, Johnson MH. Differential habituation to repeated sounds in infants at high risk for autism: NeuroReport. Published online September 2011:1. doi:10.1097/WNR.0b013e32834c0bec

26. Green SA, Hernandez L, Bookheimer SY, Dapretto M. Salience Network Connectivity in Autism Is Related to Brain and Behavioral Markers of Sensory Overresponsivity. J Am Acad Child Adolesc Psychiatry. 2016;55(7):618-626.e1. doi:10.1016/j.jaac.2016.04.013

27. Green SA, Hernandez L, Lawrence KE, et al. Distinct Patterns of Neural Habituation and Generalization in Children and Adolescents With Autism With Low and High Sensory Overresponsivity. Am J Psychiatry. 2019;176(12):1010-1020. doi:10.1176/appi.ajp.2019.18121333

28. Hudac CM, DesChamps TD, Arnett AB, et al. Early enhanced processing and delayed habituation to deviance sounds in autism spectrum disorder. Brain Cogn. 2018;123:110-119. doi:10.1016/j.bandc.2018.03.004

29. Kujala T, Aho E, Lepistö T, et al. Atypical pattern of discriminating sound features in adults with Asperger syndrome as reflected by the mismatch negativity. Biol Psychol. 2007;75(1):109-114. doi:10.1016/j.biopsycho.2006.12.007

30. Lepistö T, Kujala T, Vanhala R, Alku P, Huotilainen M, Näätänen R. The discrimination of and orienting to speech and non-speech sounds in children with autism. Brain Res. 2005;1066(1-2):147-157. 
doi:10.1016/j.brainres.2005.10.052

31. Lepistö T, Nieminen-von Wendt T, von Wendt L, Näätänen R, Kujala T. Auditory cortical change detection in adults with Asperger syndrome. Neurosci Lett. 2007;414(2):136-140. doi:10.1016/j.neulet.2006.12.009

32. Kemner, Oranje B, Verbaten MN, van Engeland H. Normal P50 Gating in Children With Autism. J Clin Psychiatry. 2002;63(3):214-217. doi:10.4088/JCP.v63n0307

33. Korpilahti P, Jansson-Verkasalo E, Mattila M-L, et al. Processing of Affective Speech Prosody is Impaired in Asperger Syndrome. J Autism Dev Disord. 2007;37(8):1539-1549. doi:10.1007/s10803006-0271-2

34. Lindström R, Lepistö-Paisley T, Vanhala R, Alén R, Kujala T. Impaired neural discrimination of emotional speech prosody in children with autism spectrum disorder and language impairment. Neurosci Lett. 2016;628:47-51. doi:10.1016/j.neulet.2016.06.016

35. Ludlow A, Mohr B, Whitmore A, Garagnani M, Pulvermüller F, Gutierrez R. Auditory processing and sensory behaviours in children with autism spectrum disorders as revealed by mismatch negativity. Brain Cogn. 2014;86:55-63. doi:10.1016/j.bandc.2014.01.016

36. Madsen GF, Bilenberg N, Jepsen JR, Glenthøj B, Cantio C, Oranje B. Normal P50 Gating in Children with Autism, Yet Attenuated P50 Amplitude in the Asperger Subcategory: Sensory Gating in Autistic Children. Autism Res. 2015;8(4):371-378. doi:10.1002/aur.1452

37. Vlaskamp C, Oranje B, Madsen GF, et al. Auditory processing in autism spectrum disorder: Mismatch negativity deficits: Auditory Processing in Autism Spectrum Disorder. Autism Res. 2017;10(11):18571865. doi:10.1002/aur.1821

38. Green SA, Hernandez L, Tottenham N, Krasileva K, Bookheimer SY, Dapretto M. Neurobiology of Sensory Overresponsivity in Youth With Autism Spectrum Disorders. JAMA Psychiatry. 2015;72(8):778-786. doi:10.1001/jamapsychiatry.2015.0737

39. Schoen S, Miller L, Green K. Pilot Study of the Sensory Over-Responsivity Scales: Assessment and Inventory. Am J Occup Ther Off Publ Am Occup Ther Assoc. 2008;62:393-406. doi:10.5014/ajot.62.4.393

40. Dunn W. Sensory Profile. Psychological Corporation; 1999.

41. Polich J. Cognitive brain potentials. Curr Dir Psychol Sci. 1993;2(6):175-179.

42. Davies PL, Chang W-P, Gavin WJ. Middle and late latency ERP components discriminate between adults, typical children, and children with sensory processing disorders. Front Integr Neurosci. 2010;4. doi:10.3389/fnint.2010.00016

43. Burack JA, Russo N, Gordon-Green C, Landry O, larocci G. Developments in the developmental approach to intellectual disability. In: Cicchetti D, ed. Handbook on Developmental Psychopathology. John Wiley \& Sons, Inc.; 2016:1-67. doi:10.1002/9781119125556

44. Burack JA, larocci G, Flanagan TD, Bowler DM. On Mosaics and Melting Pots: Conceptual Considerations of Comparison and Matching Strategies. J Autism Dev Disord. 2004;34(1):65-73. doi:10.1023/B:JADD.0000018076.90715.00 
45. Russo N, Flanagan T, larocci G, Berringer D, Zelazo PD, Burack JA. Deconstructing executive deficits among persons with autism: Implications for cognitive neuroscience. Brain Cogn. 2007;65(1):77-86. doi:10.1016/j.bandc.2006.04.007

46. Insel T, Cuthbert B, Garvey M, et al. Research Domain Criteria (RDoC): Toward a New Classification Framework for Research on Mental Disorders. Am J Psychiatry. 2010;167(7):748-751. doi:10.1176/appi.ajp.2010.09091379

47. Wechsler D, Hsiao-pin C. WASI-II: Wechsler Abbreviated Scale of Intelligence. 2nd ed. Psychological Corporation; 2011.

48. Lord C, Rutter M, DiLavore PC, Risi S, Gotham K, Bishop S. Autism Diagnostic Observation Schedule, Second Edition. Western Psychological Services,; 2012.

49. Rutter M, LeCoutier A, Lord C. The Autism Diagnostic Interview-Revised (ADI-R). Western Psychological Services; 2003.

50. Näätänen R, Gaillard AWK. 5 The Orienting Reflex and the N2 Deflection of the Event-Related Potential (ERP). In: Gaillard AWK, Ritter W, eds. Advances in Psychology. Vol 10. Tutorials in Event Related Potential Research: Endogenous Components. North-Holland; 1983:119-141. doi:10.1016/S0166-4115(08)62036-1

51. Baron-Cohen S, Wheelwright S, Skinner R, Martin J, Clubley E. The Autism-Spectrum Quotient (AQ): Evidence from Asperger Syndrome/High-Functioning Autism, Males and Females, Scientists and Mathematicians. J Autism Dev Disord. 2001;31(1):5-17. doi:10.1023/A:1005653411471

52. Tomchek SD, Dunn W. Sensory Processing in Children With and Without Autism: A Comparative Study Using the Short Sensory Profile. Am J Occup Ther. 2007;61(2):190-200. doi:10.5014/ajot.61.2.190

53. Baron-Cohen S, Hoekstra RA, Knickmeyer R, Wheelwright S. The Autism-Spectrum Quotient (AQ)Adolescent Version. J Autism Dev Disord. 2006;36(3):343-350. doi:10.1007/s10803-006-0073-6

54. Woodbury-Smith MR, Robinson J, Wheelwright S, Baron-Cohen S. Screening Adults for Asperger Syndrome Using the AQ: A Preliminary Study of its Diagnostic Validity in Clinical Practice. J Autism Dev Disord. 2005;35(3):331-335. doi:10.1007/s10803-005-3300-7

55. Perrin F, Pernier J, Bertnard O, Giard MH, Echallier JF. Mapping of scalp potentials by surface spline interpolation. Electroencephalogr Clin Neurophysiol. 1987;66(1):75-81. doi:10.1016/00134694(87)90141-6

56. Delorme A, Makeig S. EEGLAB: an open source toolbox for analysis of single-trial EEG dynamics including independent component analysis. J Neurosci Methods. 2004;134(1):9-21. doi:10.1016/j.jneumeth.2003.10.009

57. Lopez-Calderon J, Luck SJ. ERPLAB: an open-source toolbox for the analysis of event-related potentials. Front Hum Neurosci. 2014;8. doi:10.3389/fnhum.2014.00213

58. Russo N, Foxe JJ, Brandwein AB, Altschuler T, Gomes H, Molholm S. Multisensory processing in children with autism: high-density electrical mapping of auditory-somatosensory integration. Autism Res. 2010;3(5):253-267. doi:https://doi.org/10.1002/aur.152 
59. Gu X, Li Y, Yang X, Zhu Y. Spatial-Temporal Analysis of Face Processing Using an ERP Study of the Thatcher Illusion. In: 2007 29th Annual International Conference of the IEEE Engineering in Medicine and Biology Society. ; 2007:2496-2499. doi:10.1109/IEMBS.2007.4352835

60. Clayson PE, Baldwin SA, Larson MJ. How does noise affect amplitude and latency measurement of event-related potentials (ERPs)? A methodological critique and simulation study: ERP measurement. Psychophysiology. 2013;50(2):174-186. doi:10.1111/psyp.12001

61. Kiesel A, Miller J, Jolicœur P, Brisson B. Measurement of ERP latency differences: A comparison of single-participant and jackknife-based scoring methods. Psychophysiology. 2008;45(2):250-274. doi:https://doi.org/10.1111/j.1469-8986.2007.00618.x

62. Williams ZJ, Abdelmessih PG, Key AP, Woynaroski TG. Cortical Auditory Processing of Simple Stimuli is Altered in Autism: A Meta-analysis of Auditory Evoked Responses. Biol Psychiatry Cogn Neurosci Neuroimaging. Published online September 2020:S2451902220302809. doi:10.1016/j.bpsc.2020.09.011

63. Adler LE, Olincy A, Waldo M, et al. Schizophrenia, Sensory Gating, and Nicotinic Receptors. Schizophr Bull. 1998;24(2):189-202. doi:10.1093/oxfordjournals.schbul.a033320

64. Johannes S, Münte TF, Heinze HJ, Mangun GR. Luminance and spatial attention effects on early visual processing. Cogn Brain Res. 1995;2(3):189-205. doi:10.1016/0926-6410(95)90008-X

65. Billings CJ, Tremblay KL, Souza PE, Binns MA. Effects of Hearing Aid Amplification and Stimulus Intensity on Cortical Auditory Evoked Potentials. Audiol Neurotol. 2007;12(4):234-246. doi:10.1159/000101331

66. Borgmann C, Roß B, Draganova R, Pantev C. Human auditory middle latency responses: influence of stimulus type and intensity. Hear Res. 2001;158(1):57-64. doi:10.1016/S0378-5955(01)00292-1

67. Čeponienè R, Alku P, Westerfield M, Torki M, Townsend J. ERPs differentiate syllable and nonphonetic sound processing in children and adults. Psychophysiology. 2005;42(4):391-406. doi:https://doi.org/10.1111/j.1469-8986.2005.00305.x

68. Moore JK, Linthicum FH. The human auditory system: A timeline of development. Int J Audiol. 2007:46(9):460-478. doi:10.1080/14992020701383019

69. Sharma A, Kraus N, J. McGee T, Nicol TG. Developmental changes in P1 and N1 central auditory responses elicited by consonant-vowel syllables. Electroencephalogr Clin Neurophysiol Potentials Sect. 1997;104(6):540-545. doi:10.1016/S0168-5597(97)00050-6

70. Samson F, Hyde KL, Bertone A, et al. Atypical processing of auditory temporal complexity in autistics. Neuropsychologia. 2011;49(3):546-555. doi:10.1016/j.neuropsychologia.2010.12.033

71. Cheour M, Ceponiene R, Lehtokoski A, et al. Development of language-specific phoneme representations in the infant brain. Nat Neurosci. 1998;1(5):351-353. doi:10.1038/1561

72. Näätänen R. The perception of speech sounds by the human brain as reflected by the mismatch negativity (MMN) and its magnetic equivalent (MMNm). Psychophysiology. 2001;38(1):1-21. doi:https://doi.org/10.1111/1469-8986.3810001 
73. Haesen B, Boets B, Wagemans J. A review of behavioural and electrophysiological studies on auditory processing and speech perception in autism spectrum disorders. Res Autism Spectr Disord. 2011;5(2):701-714. doi:10.1016/j.rasd.2010.11.006

74. Schwartz S, Shinn-Cunningham B, Tager-Flusberg H. Meta-analysis and systematic review of the literature characterizing auditory mismatch negativity in individuals with autism. Neurosci Biobehav Rev. 2018;87:106-117. doi:10.1016/j.neubiorev.2018.01.008

75. Čeponienè R, Lepistö T, Shestakova A, et al. Speech-sound-selective auditory impairment in children with autism: They can perceive but do not attend. Proc Natl Acad Sci. 2003;100(9):5567-5572. doi:10.1073/pnas.0835631100

76. Gomot M, Giard M-H, Adrien J-L, Barthelemy C, Bruneau N. Hypersensitivity to acoustic change in children with autism: Electrophysiological evidence of left frontal cortex dysfunctioning. Psychophysiology. 2002;39(5):577-584. doi:https://doi.org/10.1111/1469-8986.3950577

77. Jansson-Verkasalo E, Ceponiene R, Kielinen M, et al. Deficient auditory processing in children with Asperger Syndrome, as indexed by event-related potentials. Neurosci Lett. 2003;338(3):197-200. doi:10.1016/S0304-3940(02)01405-2

78. Lepistö T, Kuitunen A, Sussman E, et al. Auditory stream segregation in children with Asperger syndrome. Biol Psychol. 2009;82(3):301-307. doi:10.1016/j.biopsycho.2009.09.004

79. Bertone A, Mottron L, Jelenic P, Faubert J. Enhanced and diminished visuo-spatial information processing in autism depends on stimulus complexity. Brain. 2005;128(10):2430-2441. doi:10.1093/brain/awh561

80. Ferri R, Elia M, Agarwal N, Lanuzza B, Musumeci SA, Pennisi G. The mismatch negativity and the P3a components of the auditory event-related potentials in autistic low-functioning subjects. Clin Neurophysiol. 2003;114(9):1671-1680. doi:10.1016/S1388-2457(03)00153-6

81. Russo N, Mottron L, Burack JA, Jemel B. Parameters of semantic multisensory integration depend on timing and modality order among people on the autism spectrum: Evidence from event-related potentials. Neuropsychologia. 2012;50(9):2131-2141. doi:10.1016/j.neuropsychologia.2012.05.003

82. Aykan S, Gürses E, Tokgöz-Yılmaz S, Kalaycıoğlu C. Auditory Processing Differences Correlate With Autistic Traits in Males. Front Hum Neurosci. 2020;14. doi:10.3389/fnhum.2020.584704

83. Mayer JL, Hannent I, Heaton PF. Mapping the Developmental Trajectory and Correlates of Enhanced Pitch Perception on Speech Processing in Adults with ASD. J Autism Dev Disord. 2016;46(5):15621573. doi:10.1007/s10803-014-2207-6

84. Senkowski D, Saint-Amour D, Höfle M, Foxe JJ. Multisensory interactions in early evoked brain activity follow the principle of inverse effectiveness. Neurolmage. 2011;56(4):2200-2208. doi:10.1016/j.neuroimage.2011.03.075

85. Burack JA, Russo N, Kovshoff H, et al. How I Attend-Not How Well Do I Attend: Rethinking Developmental Frameworks of Attention and Cognition in Autism Spectrum Disorder and Typical Development. J Cogn Dev. 2016;17(4):553-567. doi:10.1080/15248372.2016.1197226 
86. Plaisted K, Swettenham J, Rees L. Children with Autism Show Local Precedence in a Divided Attention Task and Global Precedence in a Selective Attention Task. J Child Psychol Psychiatry. 1999;40(5):733-742. doi:https://doi.org/10.1111/1469-7610.00489

87. Schönbrodt FD, Perugini M. At what sample size do correlations stabilize? J Res Personal. 2013;47(5):609-612. doi:10.1016/j.jrp.2013.05.009

88. Baranek GT. Efficacy of Sensory and Motor Interventions for Children with Autism. J Autism Dev Disord. 2002;32(5):26.

\section{Figures}



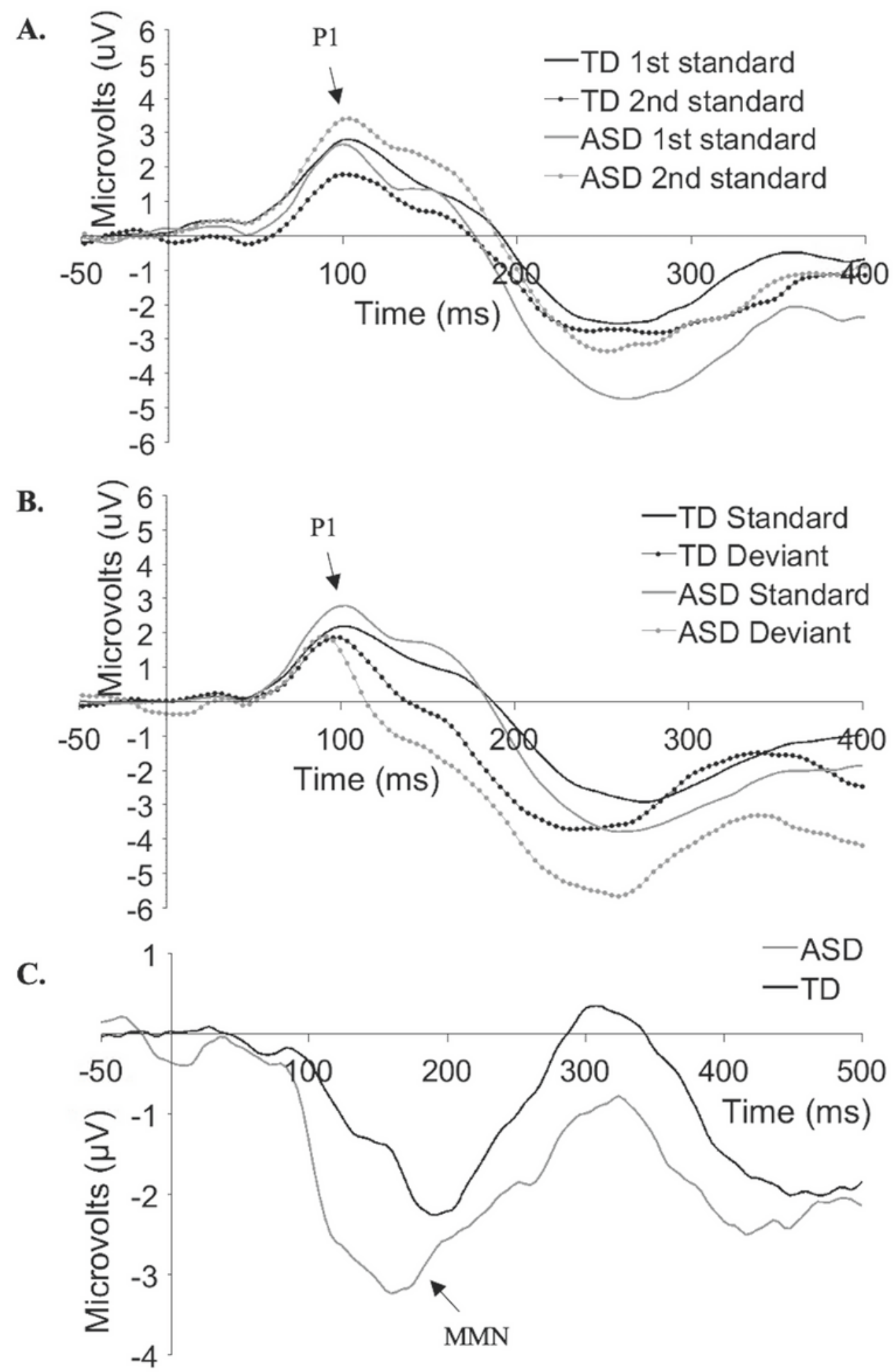

\section{Figure 1}

Response-locked ERP waveforms at the FCz electrode site for Autism Spectrum Disorder (ASD) and TD children during the oddball task, corresponding to (A) P1 habituation, comparing the 1st to 2nd successive standard, (B) P1 discrimination, comparing the standard to the deviant stimuli, and (C) MMN discrimination from subtracting the standard from the deviant grand average waveforms. 


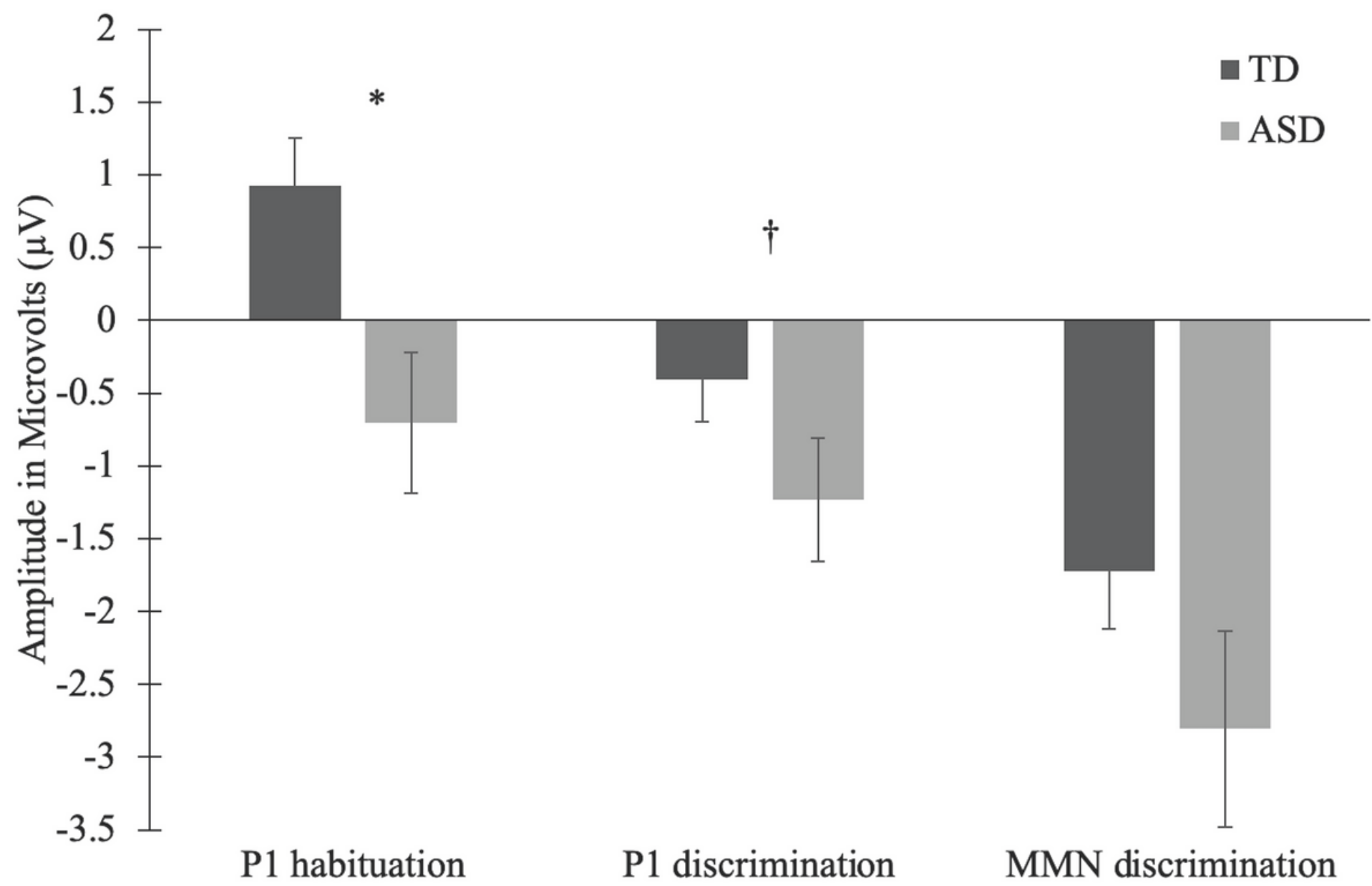

Figure 2

P1 habituation (amplitude of 1st minus 2nd standard) and P1 discrimination (amplitude of deviant minus standard) analyzed by group $(95 \% \mathrm{Cl})$. 


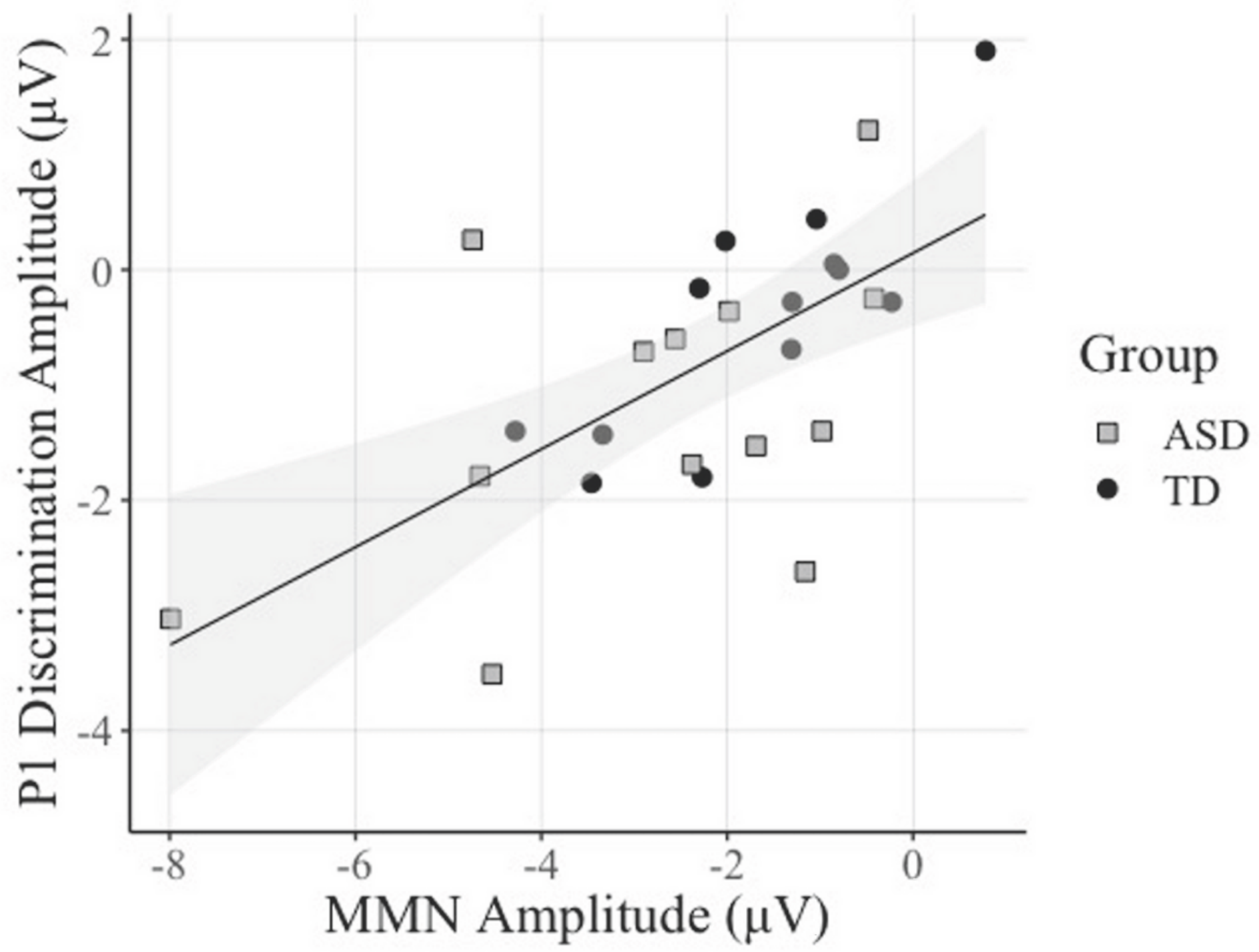

Figure 3

A significant positive correlation between the MMN amplitude and P1 discrimination amplitude. 
A.

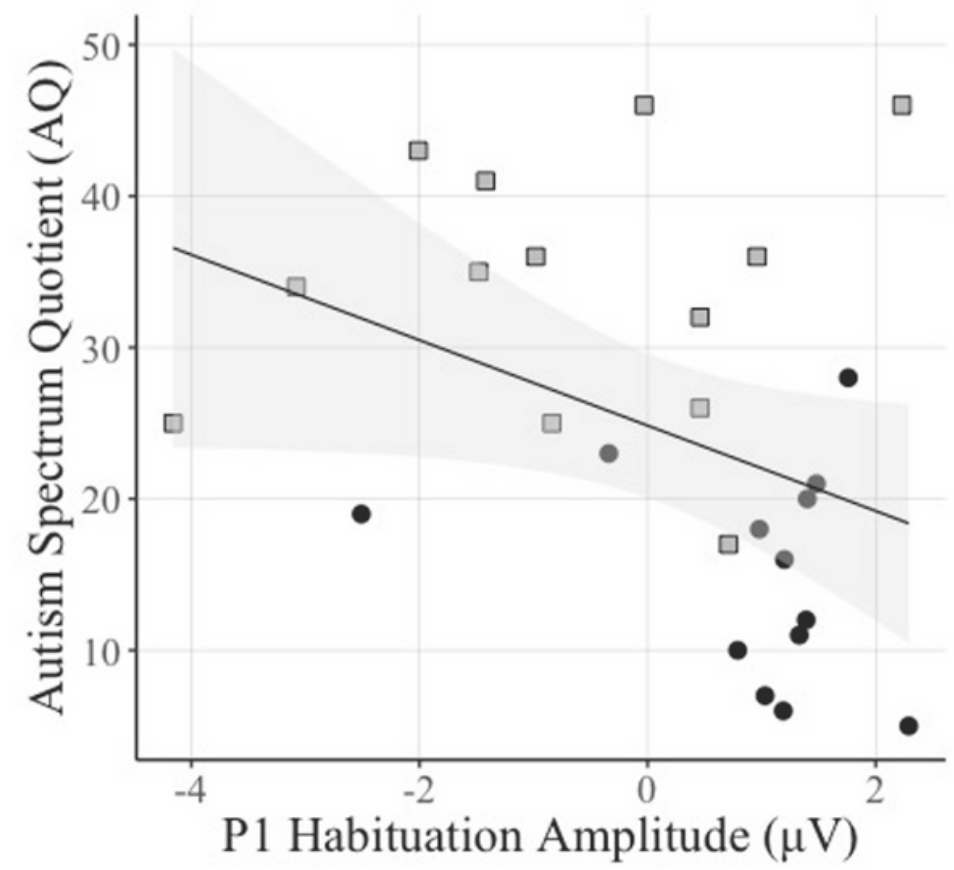

C.

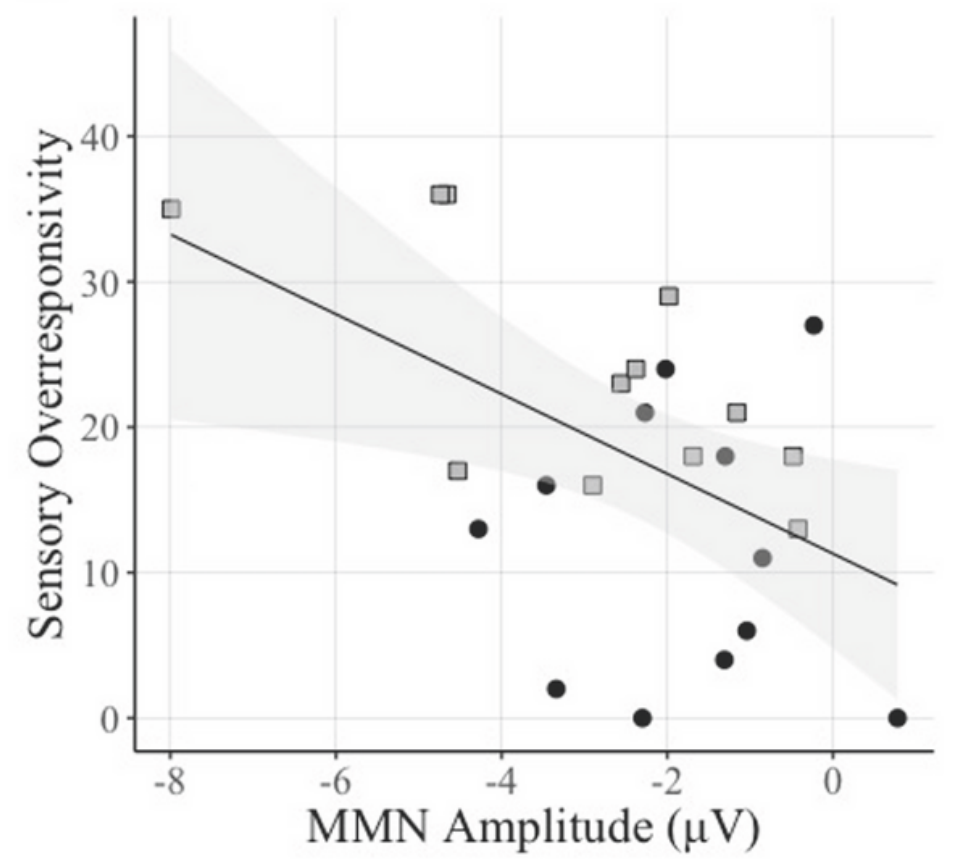

B.

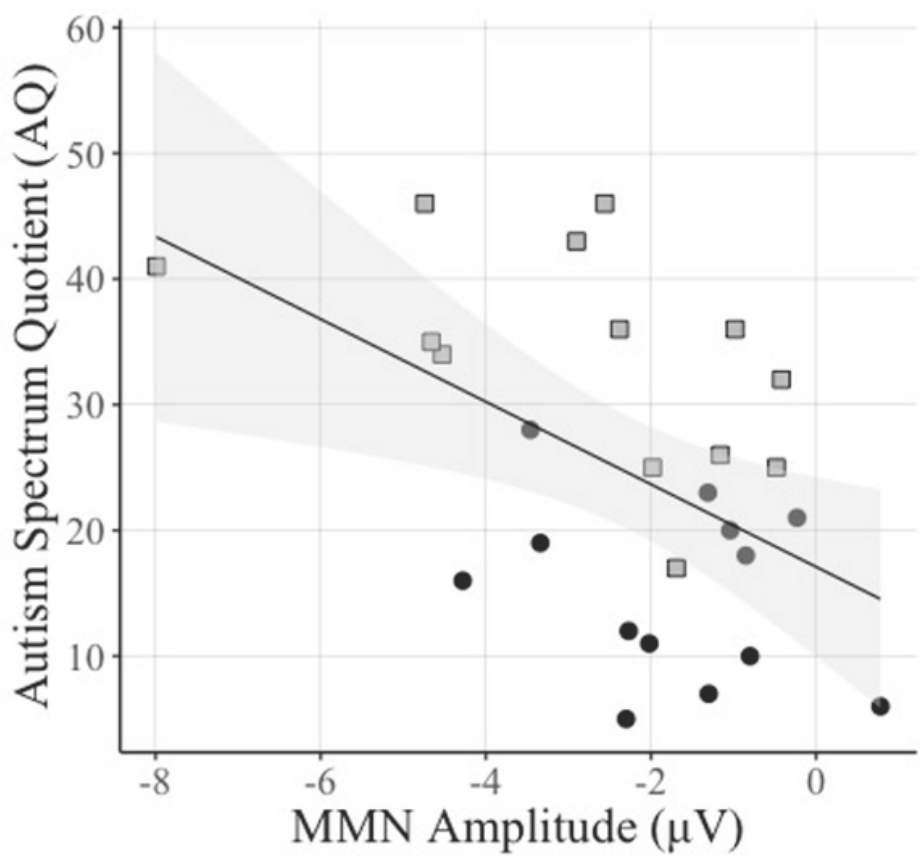

Group

$\square$ ASD

- TD

Figure 4

Correlations between the $\mathrm{AQ}$ and $\mathrm{A}$ ) $\mathrm{P} 1$ habituation amplitude and $\mathrm{B}$ ) $\mathrm{MMN}$ amplitude, and C) between Sensory Overresponsivity and the MMN, by group. 